\title{
Cepstrum Analysis and Fault Diagnosis of Combine Harvester Transaxle
}

\author{
Bo Yu ${ }^{a}$, Qiang Wang ${ }^{b}$, Shuang Wang, Min Liao, Jun Yi \\ School of Mechanical Engineering, Xihua University, Chengdu 610039, China \\ a540082120@qq.com, bwq_py123@163.com
}

Keywords: combine harvester, cepstrum analysis, transaxle, fault diagnosis, vibration signal.

\begin{abstract}
Head-feed combine harvester transaxle transmits motive force to the header and driving system, with adverse working conditions transaxle is especially prone to failure. It is not easy to detective and recognize faults of transaxle with traditional diagnostic methods as "Listening, Observation and Touch", because it has complex inner structures, especially number of meshing gears. In order to find fault components and distinguish failure types, analyzing the vibration signal of the transaxle. In the present paper, vibration signals of a certain type head-feed combine harvester transaxle are extracted by SENDIG S956Y vibration analyzer; fault shaft (frequency is 6.34Hz) and meshing gears (quefrency is 3.9ms) of the transaxle are located by cepstrum and the zoom amplitude spectrum and failure types of fault shaft and meshing gears are diagnosed out based on the exclusive characteristics of vibration signal of different faults in rotating machine. The results show that cepstrum and the zoom amplitude spectrum approaches that are proposed in the paper are successfully to ascertain the combine harvester transaxle fault.
\end{abstract}

\section{Introduction}

Transaxle is a key part of the combine harvester drive system, it has a great influence on harvest operation. Once a serious fault or damage occur in transaxle, the combine harvester has to stop harvesting and to repair it, and great economic loss would be caused. It is very significance to improve the economic performance and avoid having a major accident when diagnosing and dealing with a fault before it becomes serious.

Statistics show that the highest failure proportion in all kinds of transmission is gear, about $60 \%$. At present, extracting the fault information from the gear vibration signal and noise signal (especially vibration signal) is the effective way of gearbox fault monitoring. The existing methods of fault diagnosis include the power spectrum analytic method, side-band algorithm and the cepstrum analysis, etc. ${ }^{[1]}$ The effectiveness of autocorrelation and cepstrum analyses in detection and diagnosis of rolling bearing has been shown in [2]. In order to ascertain the gear fault, in [3] both the Wigner-Viller distribution and Fourier transformation cepstrum have been used to locate the fault. [4] has proposed an experimental sideband algorithm for an automatic wind turbine gear tooth fault detection and diagnosis. The method of aero-engine rubbing positions identification based on cepstrum analysis has been proposed in [5]. In order to monitor and maintain a turbine gearbox in time, a method to diagnose turbine gearbox gearcrack based on wavelet packet and cepstrum analysis has been proposed in [6].

Cepstrum analysis has been validated in gearbox fault diagnosis of aviation, oil equipment and wind turbine, etc. Because of number of meshing gears in the transaxle, when gears mesh at the same time will produce cluster of side band spectrum in spectrogram. Traditional Fourier transform is difficult to distinguish the fault signal, but cepstrum analysis can reduce the cluster of side band spectrum to single lines, and then easier to identify cycle structure of complex spectrogram. ${ }^{[7,8]}$ Therefore, cepstrum and the zoom amplitude spectrum approaches are proposed for fault diagnosis and analysis of combine harvester transaxle. 


\section{The principle of cepstrum}

In 1962, the cepstrum analysis was put forward by Bogert, Healy and Tukey, etc. The failure frequencies hide in multiple clusters modulation frequency can be decomposed and recognized through cepstrum analysis. The cepstrum transformation is actually the Fourier integral transformation of frequency domain signals transform again. ${ }^{[9]}$

The measured vibration signal $x(t)$ output signal $y(t)$ through a system impulse response function $h(t)$. Output signal $y(t)$ is equal to the convolution of $x(t)$ and $h(t)$ :

$y(t)=x(t) * h(t)$

The equation of power cepstrum:

$$
C_{p}(\tau)=\left\{F^{-1}\left[\lg |X(f)|^{2}\right]\right\}^{2}=\left\{F^{-1}\left[\lg S_{x}(f)\right]\right\}^{2}
$$

In the equation (2), $X(f)$ and $S_{x}(f)$ is the Fourier transform and the power spectral density function of $x(t)$ respectively. The square root of the equation (2) called amplitude cepstrum is commonly used in engineering:

$$
C_{x}(\tau)=F^{-1}\left[\lg S_{x}(f)\right]
$$

The convolution of $x(t)$ and $h(t)$ in the equation (1) can be reduced to simple superposition by using the cepstrum technology:

$$
C_{y}(\tau)=C_{x}(\tau)+C_{h}(\tau)
$$

Extracting signal components through the cepstrum transformation to achieve the purpose of fault diagnosis. The basic steps of cepstrum analysis of signals is shown in figure 1 .

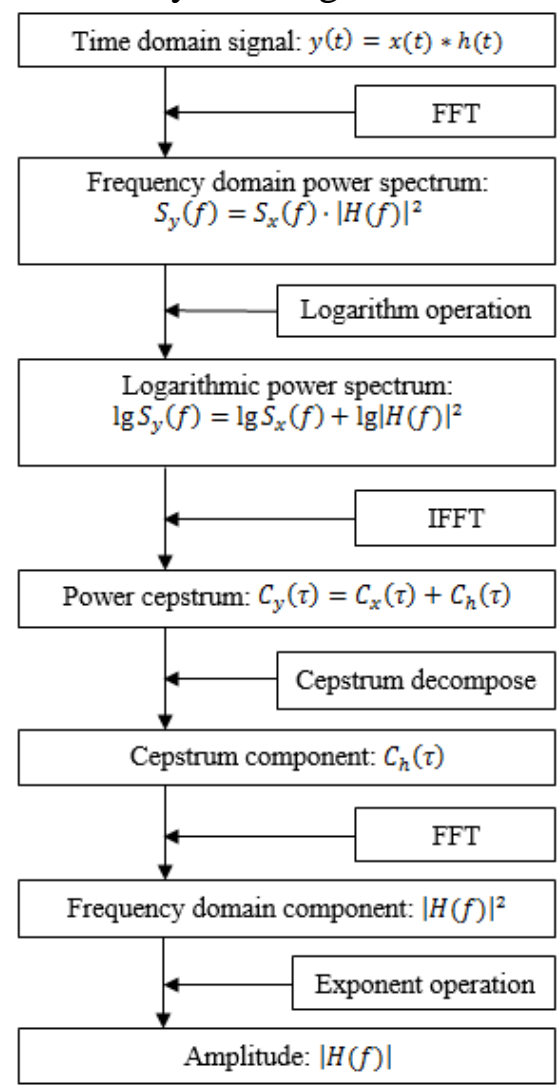

Fig. 1 The basic steps of cepstrum analysis of signals

\section{The cepstrum analysis of transaxle vibration signal}

A certain type head-feed combine harvester transaxle was heard abnormal sound when doing running-in test on the bench, which is designed by our project group and manufactured by a harvester 
manufacturing company of Sichuan. In order to locate the fault component with abnormal sound, cepstrum analysis was carried out on transaxle vibration signal.

The transaxle combines with HST (Hydro Static Transmission), transmits motive force to the header and driving system. There are two independent gear transmission chain in the transaxle: the gear transmission chain of the header and the gear transmission chain of the driving system. The gear transmission chain of the header was heard abnormal sound when doing running-in test. So only fault diagnosis of the gear transmission chain of the header was taken in this paper. The gear transmission chain of the header is shown in figure 2.

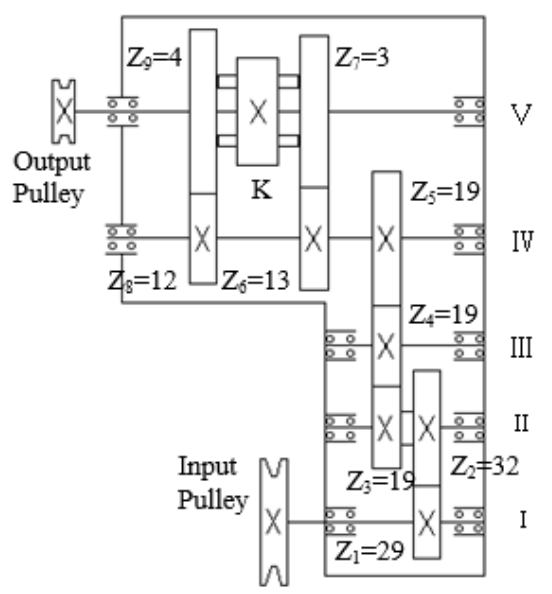

Fig. 2 The gear transmission chain of the header

Axis $I$ is the input shaft, with the rotating speed $n_{1}=1400$ rpm. The number of gear teeth respectively: $Z_{1}=29, Z_{2}=32, Z_{3}=19, Z_{4}=19, Z_{5}=19, Z_{6}=13, Z_{7}=31, Z_{8}=12, Z_{9}=40$. Axis $V$ is the output shaft which can outputs different rotating speed by sliding the clutch gear $K$ to mesh $Z_{6}$ and $Z_{7}$ or $\mathrm{Z}_{8}$ and $\mathrm{Z}_{9}$. The rotational frequencies (quefrencies) and meshing frequencies (quefrencies) of the transaxle are shown in table 1.

Table 1 The rotational/meshing frequencies (quefrencies) of the transaxle

\begin{tabular}{ccc}
\hline & Frequency $(\mathrm{Hz})$ & Quefrency $(\mathrm{ms})$ \\
\hline Axis I & 23.33 & 43 \\
Axis II & 21.14 & 47 \\
Axis III & 21.14 & 47 \\
Axis IV & 21.14 & 47 \\
Axis V & $6.34 / 8.87$ & $158 / 113$ \\
$\mathrm{Z}_{1} / \mathrm{Z}_{2}$ & 676.57 & 1.5 \\
$\mathrm{Z}_{3} / \mathrm{Z}_{4}$ & 401.66 & 2.5 \\
$\mathrm{Z}_{4} / \mathrm{Z}_{5}$ & 401.66 & 2.5 \\
$\mathrm{Z}_{6} / \mathrm{Z}_{7}$ & 274.82 & 3.6 \\
$\mathrm{Z}_{8} / \mathrm{Z}_{9}$ & 253.68 & 3.9 \\
\hline
\end{tabular}

The SENDIG S956Y vibration analyzer was used to detect vertical vibration signals of the input shaft $\mathrm{I}$ and output shaft $\mathrm{V}$. The layout of measuring points on field test is shown in figure 3.

The zoom amplitude spectrum of signals were detected from the input shaft I and output shaft $\mathrm{V}$ is shown in (a) and (b) of figure 4. Can be seen in the figure (a), the amplitude of the frequencies: $2.5 \mathrm{~Hz}, 7.5 \mathrm{~Hz}, 12.5 \mathrm{~Hz}, 25 \mathrm{~Hz}, 32.5 \mathrm{~Hz}$ fluctuate significantly. In the figure (b) the amplitude of the frequencies: $2.5 \mathrm{~Hz}, 7.5 \mathrm{~Hz}, 15 \mathrm{~Hz}, 22.5 \mathrm{~Hz}, 30 \mathrm{~Hz}$ fluctuate significantly. Two groups of frequencies are similar to the rotating frequency and frequency doubling of the output shaft $\mathrm{V}(6.34 \mathrm{~Hz})$, so that proves that shaft $\mathrm{V}$ has failure symptoms. Contrasting figure (a) and (b), the abnormal vibration of 
shaft $\mathrm{V}$ can be deduced. Judging shaft $\mathrm{V}$ has bending faults of shaft and partial abrasion based on the exclusive characteristics of vibration signal of different faults in rotating machine.
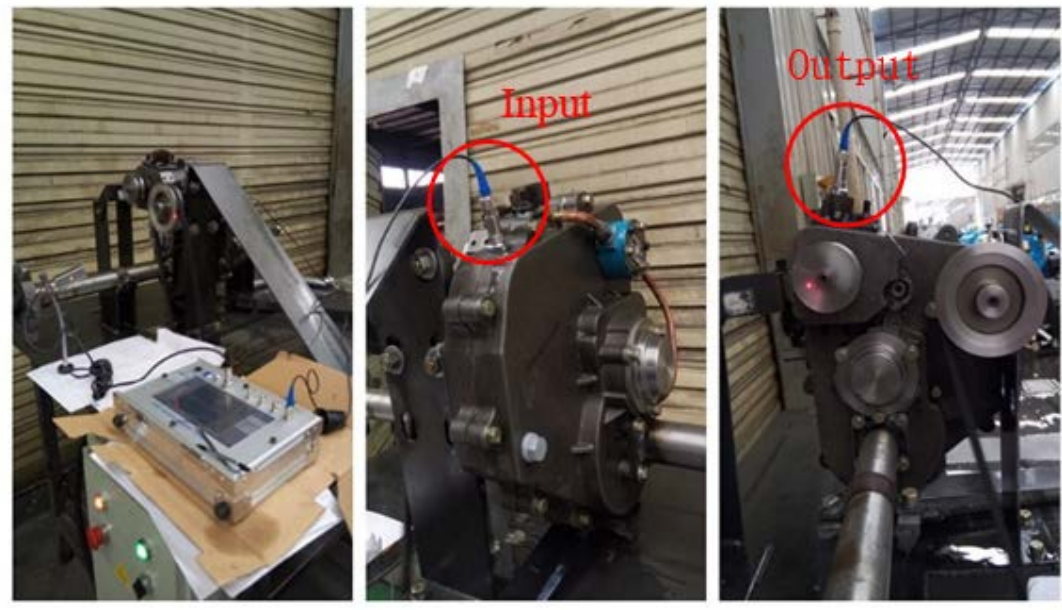

Fig. 3 The layout of measuring points on field test

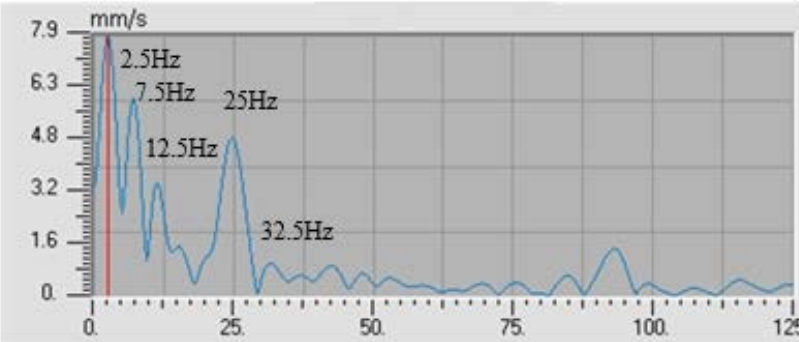

$\begin{array}{cll}\text { Frequency } & \text { Peak } & \% \\ 2.5 & 8.67 & 100 \\ 7.5 & 6.25 & 72.12 \\ 25 . & 5.26 & 60.65 \\ 125 & 27 & 31.19 \\ 925 & 1.54 & 17.82 \\ 42.5 & 1.03 & 11.9 \\ 32.5 & 1.01 & 11.62 \\ 47.5 & 0.75 & 8.6 \\ 37.5 & 0.71 & 819 \\ 85 . & 0.69 & 8.01\end{array}$

(a)

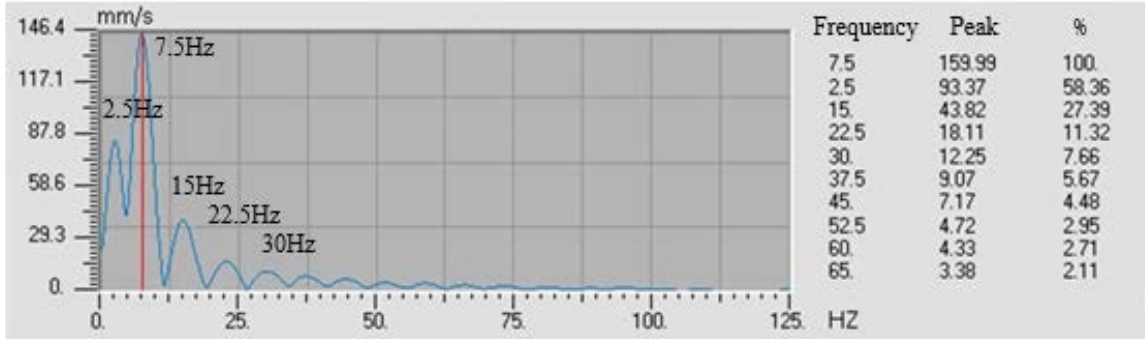

(b)

Fig. 4 The zoom amplitude spectrum

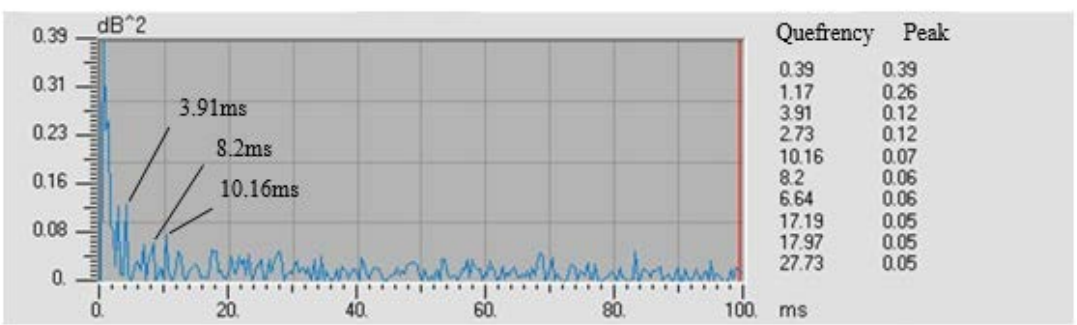

(a)

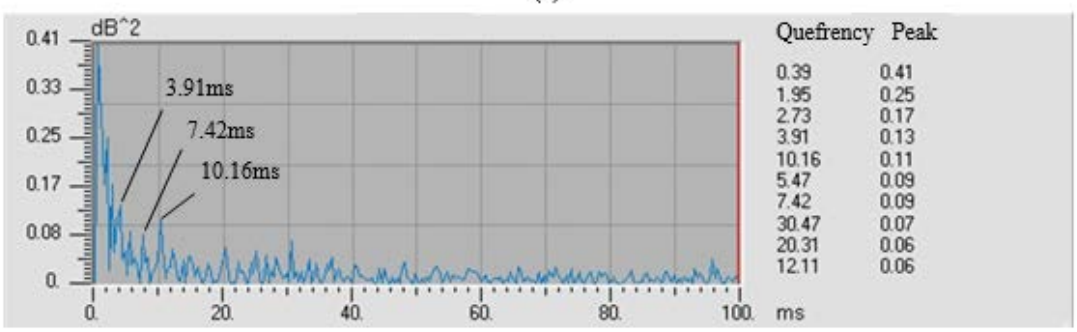

(b)

Fig. 5 The cepstrum of meshing gears 
The cepstrum of signals were detected from the input shaft $\mathrm{I}$ and output shaft $\mathrm{V}$ is shown in (a) and (b) of figure 5. The cepstrum of figure (a) is basically same as (b), this just reflects a major advantage of the cepstrum: it is not sensitive to signal transmission path and measuring position, and this insensitivity is benefit to observe the characteristic frequency and resonant frequency. The cepstrum components are seen in figure (a): $3.91 \mathrm{~ms}, 8.2 \mathrm{~ms}$ and $10.16 \mathrm{~ms}$, or in figure (b): $3.91 \mathrm{~ms}$, $7.42 \mathrm{~ms}$ and $10.16 \mathrm{~ms}$, are similar to the quefrency, second and third quefrency of the meshing gears $\mathrm{Z}_{8} / \mathrm{Z}_{9}$ (3.9ms). So it proves the meshing gears $\mathrm{Z}_{8} / \mathrm{Z}_{9}$ have faults.

Shaft $\mathrm{V}$ is diagnosed with bending faults of shaft and partial abrasion by analyzing figure 4 , and faults of the meshing gears $Z_{8} / Z_{9}$ are located by cepstrum. Judging the meshing gears $Z_{8} / Z_{9}$ have localized wear based on the exclusive characteristics of cepstrum of gear vibration signal. Finding Shaft $\mathrm{V}$ has a bending deformation symptom and the meshing gears $\mathrm{Z}_{8} / \mathrm{Z}_{9}$ have localized wear when stop to checking. So the diagnosis results have been verified.

\section{Conclusion}

In this paper, the cepstrum analysis has been used in fault diagnosis of the head-feed combine harvester transaxle based on the spectrum characteristics of gear vibration. Faults of the transaxle have been located by cepstrum and the zoom amplitude spectrum approaches, and the axis (V) and gears $\left(Z_{8}\right.$ and $\left.Z_{9}\right)$ with faults have been found out. Failure types of fault shaft and meshing gears have been diagnosed out based on the exclusive characteristics of vibration signal of different faults in rotating machine. The results show that cepstrum and the zoom amplitude spectrum approaches are successfully to ascertain the combine harvester transaxle fault.

\section{Acknowledgment}

This work was financially supported by the Innovation Fund of Xihua University Postgraduated (ycjj2014058), the Research Project of Application Basis of Sichuan Province (2014JY0055), the Research Project of Fluid and Machinery of the key Laboratory of The Ministry of Education (szjj2014-048), the Science and Technology Support Project of Sichuan Province (2015NZ0055).

\section{References}

[1]. Zhang Jian, Mechanical Fault Diagnosis Technology [M]. Beijing: CHINA MACHINE PRESS, 2014, p.152-153.

[2]. El Morsy, M.; Achtenová, G., Rolling Bearing Fault Diagnosis Techniques - Autocorrelation and Cepstrum Analyses [J]. IEEE/23rd Mediterranean Conference on Control and Automation (MED). June 16-19, 2015, Torremolinos, Spain, p.328-334.

[3]. Pi Jun, Yan Guo-hua, Wang Xuan, et al. Gear Fault Detection with Wigner-Viller Distribution Based Cepstrum Approach [J]. IEEE/2nd International Conference on Computer Engineering and Technology (ICCET). April 16-18, 2010, Chengdu, p.V1-500-V1-502.

[4]. Zappalá, D.; Tavner, P.J.; Crabtree, C.J.; et al. Side-band algorithm for automatic wind turbine gearbox fault detection and diagnosis [J]. Renewable Power Generation, IET. Vol. 8 (2014) No. 4, p.380-389.

[5]. Chen Guo, Yu Mingyue, Liu Yongquan, et al. Identifying Rotor-stator Rubbing Positions Using the Cepstrum Analysis Technique [J]. JOURNAL OF MECHANICAL ENGINEERING. Vol. 50 (2014) No. 7, p.32-38.

[6]. Luo Yi, Zhen Li-jing. Diagnosis method of turbine gearbox gearcrack based on wavelet packet and cepstrum analysis [J]. JOURNAL OF VIBRATION AND SHOCK. Vol. 34 (2015) No. 3, p.210-214. 
[7]. Li Xiaohu, Jia Minping, Xu Feiyun. Spectrum Analysis and Its Application to Gearbox Fault Diagnosis [J]. Journal of Vibration, Measurement \& Diagnosis. Vol. 23 (2003) No. 3, p.168-171.

[8]. Wang Jiangping. The Application of the Mechanical Equipment Fault Diagnosis Technology [M]. XI'an: Northwestern Polytechnical University Press, 2001, p.41-43.

[9]. Zhang Jin, Zhang Yaohui, Huang Manguo. The Cepstrum and Its Application in Gear Box Diagnosis [J]. MECHANICAL ENGINEER. 2005 No. 8, p.34-36. 